\title{
Kinetic theory with spin: From massive to massless fermions
}

\author{
Xin-Li Sheng®, ${ }^{1}$ Qun Wang, ${ }^{1}$ and Xu-Guang Huang ${ }^{2,3}$ \\ ${ }^{1}$ Interdisciplinary Center for Theoretical Study and Department of Modern Physics, \\ University of Science and Technology of China, Hefei, Anhui 230026, China \\ ${ }^{2}$ Physics Department and Center for Particle Physics and Field Theory, \\ Fudan University, Shanghai 200433, China \\ ${ }^{3}$ Key Laboratory of Nuclear Physics and Ion-beam Application (MOE), \\ Fudan University, Shanghai 200433, China
}

(Received 10 May 2020; accepted 12 July 2020; published 23 July 2020)

\begin{abstract}
We find that the recently developed kinetic theories with spin for massive and massless fermions are smoothly connected. By introducing a reference-frame vector, we decompose the dipole-moment tensor into electric and magnetic dipole moments. We show that the axial-vector component of the Wigner function contains a contribution from the transverse magnetic dipole moment, which accounts for the transverse spin degree of freedom (d.o.f.) and vanishes smoothly in the massless limit. As a result, the kinetic equations, describing 4 d.o.f. for massive fermions, smoothly becomes the chiral kinetic equations describing 2 d.o.f. in the massless limit. We also confirm the small-mass behavior of the Wigner function by an explicit calculation using a Gaussian wave packet.
\end{abstract}

DOI: 10.1103/PhysRevD.102.025019

\section{INTRODUCTION}

In noncentral heavy-ion collisions, a large orbital angular momentum (OAM) [1-3] as well as a very strong electromagnetic field [4-10] are generated. Part of the OAM is transferred to the hot and dense matter or the quark-gluon plasma in the form of vorticity fields [11-14] and leads to a global spin polarization perpendicular to the reaction plane [1,15-20]. The global spin polarization of $\Lambda$ hyperons has been observed by the STAR Collaboration in $\mathrm{Au}+\mathrm{Au}$ collisions at $\sqrt{s_{N N}}=7.7-200 \mathrm{GeV}[21,22]$; see, e.g., Refs. [23-25] for recent reviews. The interaction between the strong magnetic field and fermion spin leads to the chiral magnetic effect (CME) [26-28], which can probe the topological fluctuation of quantum chromodynamics vacuum. The search for the CME is one of the major efforts in experiments of heavy ion collisions; see, e.g., Refs. [29-34] for reviews.

For massless fermions with definite helicity, the chiral kinetic theory (CKT) is a useful tool to describe the chiral effects in phase space [35-48]. The Lorentz invariance for chiral fermions is proved to be nontrivial: the side-jump effect appears to ensure the conservation of the total angular momentum in binary collisions [49-51]. Recent

Published by the American Physical Society under the terms of the Creative Commons Attribution 4.0 International license. Further distribution of this work must maintain attribution to the author(s) and the published article's title, journal citation, and DOI. Funded by SCOAP . numerical simulation [52] shows that the side-jump effect may provide a possible explanation of the puzzle of the $\Lambda$ 's local spin polarization $[53,54]$.

In reality, all quarks have masses. Although the masses of up and down quarks are small compared to the typical temperature of the quark-gluon plasma, the mass of strange quark is not. The massive strange quarks play an essential role in the $\Lambda$ 's spin polarization as well as in the spin alignments of $\phi$ or $K^{* 0}$ mesons described by the 00 component of the spin density matrix [55-57]. Therefore, a kinetic theory for massive fermions with spin, also called the spin kinetic theory, is required to describe the spin evolution of massive quarks in phase space. Such a theory was constructed many years ago for nonrelativistic dilute spinful gases [58,59] and has recently been formulated for relativistic Dirac fermions using the covariant Wigner functions [60-64] and equal-time Wigner functions [65]. It can also be constructed in the worldline formalism [66,67].

However, there are fundamental differences between symmetries of massive and massless fermions. In 1939, Wigner proposed the concept of the little group [68], the group that leaves a particle's four-momentum invariant. For a massive particle, the little group is the rotational group $O(3)$, which is associated with the spin in the particle's rest frame. Wigner also showed that the little group for the massless particle is the two-dimensional Euclidean group $E(2)$. The rotational degree of freedom of $E(2)$ corresponds to the helicity, while the 2 translational degrees of freedom correspond to the gauge symmetry of the massless particle 
[68-70]. It can be proved that the $E(2)$ group can be obtained as the infinite-boost limit or massless limit of the $O$ (3) group [69]. However, how the kinetic theories for massive fermions and massless fermions are connected under such a limiting process is unclear. There are proposals to make a smooth transition between the two kinetic theories [61,62], but these proposals are based on the assumption that the spin polarization or dipole-moment tensor for massive fermions can be smoothly reduced to its massless forms when $m \rightarrow 0$. This assumption has not been justified.

In this paper, we propose a kinetic theory with spin in the Wigner function formalism that can smoothly transit between the massive and massless cases. The main idea is to project out the transverse-spin contributions in the kinetic theory and to show that they smoothly vanish in the massless limit. In such a way, two of the four kinetic equations in the massive case become irrelevant in the massless limit, leaving the other two to constitute the CKT that describes the vector- and axial-charge distributions. The paper is organized as follows. In Sec. II, we briefly review the Wigner function formalism for massive fermions up to the linear order in $\hbar$. Then in Sec. III, to connect with the massless case, we introduce a reference frame in the massive case. The dipole-moment tensor and spinpolarization vector are expressed in terms of referenceframe dependent quantities such as the fermion number density, the magnetic dipole moment, and the axial-charge density. In Sec. IV, the ensemble of particle states of the Wigner function is replaced by the one-particle wave packet state with finite momentum and space-time dispersion. In this case, we derive explicit expressions for the fermion number density, the magnetic dipole moment, and the axialcharge density and obtain their small-mass behaviors. In Sec. V, the Wigner-function components as well as the kinetic equations are written in forms with mass corrections explicitly singled out. Turning off the mass corrections, we can recover the massless case smoothly. A summary of the results is given in the final section.

Throughout this paper, we use the units $c=k_{B}=1$ but keep the reduced Planck's constant $\hbar$ explicitly. The electromagnetic potential is labeled by $\mathbb{A}^{\mu}$ with the electric charge being absorbed. This means that we can recover the electric charge $Q$ of the fermion by replacing $\mathbb{A}^{\mu} \rightarrow Q \mathbb{A}^{\mu}$. We consider the background spacetime to be Minkowskian, but all the calculations can be similarly carried out in curved spacetime as well (the kinetic theories for massive and massless fermions in curved spacetime were studied in Refs. [43,63]).

\section{WIGNER FUNCTION UP TO LINEAR ORDER IN $\hbar$}

We define the covariant Wigner function in an external classical electromagnetic field or U(1) gauge field as the Fourier transform of the two-point correlation function,

$$
\begin{aligned}
W(x, p) \equiv & \int \frac{d^{4} y}{(2 \pi \hbar)^{4}} e^{-\frac{i}{\hbar} p \cdot y} U\left(x+\frac{y}{2}, x-\frac{y}{2}\right) \\
& \times\left\langle\Omega\left|\hat{\bar{\psi}}\left(x+\frac{y}{2}\right) \otimes \hat{\psi}\left(x-\frac{y}{2}\right)\right| \Omega\right\rangle,
\end{aligned}
$$

where the tensor product is defined as $[A \otimes B]_{i j}=A_{j} B_{i}$ with $i=1-4$ the Dirac index. Here, $\langle\Omega|\hat{O}| \Omega\rangle$ represents the expectation value of the operator $\hat{O}$ on a given quantum state $|\Omega\rangle$, and the gauge link $U\left(x+\frac{y}{2}, x-\frac{y}{2}\right)$ is defined in a straight line,

$U\left(x+\frac{y}{2}, x-\frac{y}{2}\right) \equiv \exp \left[-\frac{i}{\hbar} y^{\mu} \int_{-1 / 2}^{1 / 2} d s \mathbb{A}_{\mu}(x+s y)\right]$,

with $\mathbb{A}_{\mu}$ being the U(1) gauge potential. For a classical background field, the gauge link is purely a phase factor instead of an operator. In order to clearly display the physical meaning of the Wigner function, it is advantageous to decompose $W(x, p)$ in terms of 16 independent generators of the Clifford algebra, $\left\{1, i \gamma^{5}, \gamma^{\mu}, \gamma^{5} \gamma^{\mu}, \sigma^{\mu \nu}\right\}$, where 1 is the $4 \times 4$ unit matrix, $\gamma^{5} \equiv i \gamma^{0} \gamma^{1} \gamma^{2} \gamma^{3}$, and $\sigma^{\mu \nu} \equiv \frac{i}{2}\left[\gamma^{\mu}, \gamma^{\nu}\right]$

$W=\frac{1}{4}\left(\mathcal{F}+i \gamma^{5} \mathcal{P}+\gamma^{\mu} \mathcal{V}_{\mu}+\gamma^{5} \gamma^{\mu} \mathcal{A}_{\mu}+\frac{1}{2} \sigma^{\mu \nu} \mathcal{S}_{\mu \nu}\right)$.

According to the parities and properties under a Lorentz transformation, the coefficients $\mathcal{F}, \mathcal{P}, \mathcal{V}_{\mu}, \mathcal{A}_{\mu}$, and $\mathcal{S}_{\mu \nu}$ are called the scalar, pseudoscalar, vector, axial-vector, and tensor components of the Wigner function. They are identified as the densities of some physical quantities in phase space [71]. For example, $\mathcal{V}_{\mu}$ gives the fermion vector current density, $\mathcal{A}_{\mu}$ gives the axial current or spin density, and $\mathcal{S}_{\mu \nu}$ gives the electric/magnetic dipole-moment density.

Applying the Dirac equation to the Wigner function, one can derive the equation of motion for the Wigner function. The Wigner-function equation of motion has analytical solutions in some special cases, such as in a constant electromagnetic field. In a general space-time dependent electromagnetic field, the semiclassical expansion in the reduced Planck's constant $\hbar$ provides a powerful method to solve out the Wigner function order by order in $\hbar$. The Wigner function at the lowest or zeroth order in $\hbar$ is independent of spin, while at the first order in $\hbar$, the Wigner function contains the spin degrees of freedom. In this paper, we only consider the Wigner function up to $\mathcal{O}(\hbar)$. The most general solution up to this order is given in Ref. [61], 


$$
\begin{aligned}
\mathcal{F} & =m\left[V \delta\left(p^{2}-m^{2}\right)-\frac{\hbar}{2} F^{\alpha \beta} \Sigma_{\alpha \beta} \delta^{\prime}\left(p^{2}-m^{2}\right)\right]+\mathcal{O}\left(\hbar^{2}\right), \\
\mathcal{P} & =\frac{\hbar}{4 m} \epsilon^{\mu \nu \alpha \beta} \nabla_{\mu}\left[p_{\nu} \Sigma_{\alpha \beta} \delta\left(p^{2}-m^{2}\right)\right]+\mathcal{O}\left(\hbar^{2}\right), \\
\mathcal{V}^{\mu} & =p^{\mu}\left[V \delta\left(p^{2}-m^{2}\right)-\frac{\hbar}{2} F^{\alpha \beta} \Sigma_{\alpha \beta} \delta^{\prime}\left(p^{2}-m^{2}\right)\right]+\frac{\hbar}{2} \nabla_{\nu}\left[\Sigma^{\mu \nu} \delta\left(p^{2}-m^{2}\right)\right]+\mathcal{O}\left(\hbar^{2}\right), \\
\mathcal{A}^{\mu} & =n^{\mu} \delta\left(p^{2}-m^{2}\right)+\hbar \tilde{F}^{\mu \nu} p_{\nu} V \delta^{\prime}\left(p^{2}-m^{2}\right)+\mathcal{O}\left(\hbar^{2}\right), \\
\mathcal{S}^{\mu \nu} & =m\left[\Sigma^{\mu \nu} \delta\left(p^{2}-m^{2}\right)-\hbar F^{\mu \nu} V \delta^{\prime}\left(p^{2}-m^{2}\right)\right]+\mathcal{O}\left(\hbar^{2}\right),
\end{aligned}
$$

where the dipole-moment tensor satisfies the following constraint equation:

$$
\delta\left(p^{2}-m^{2}\right)\left[p_{\nu} \Sigma^{\mu \nu}-\frac{\hbar}{2} \nabla^{\mu} V\right]=\mathcal{O}\left(\hbar^{2}\right),
$$

with $\nabla^{\mu} \equiv \partial_{x}^{\mu}-F^{\mu \nu} \partial_{p \nu}$. The spin polarization vector $n^{\mu}$ can be expressed by the dipole-moment tensor $\Sigma^{\mu \nu}$ as follows:

$$
n^{\mu}=-\frac{1}{2} \epsilon^{\mu \nu \alpha \beta} p_{\nu} \Sigma_{\alpha \beta} .
$$

The undetermined functions $V$ and $n^{\mu}$ satisfy the generalized Boltzmann equation and the generalized BargmannMichel-Telegdi (BMT) equation, respectively,

$$
\begin{aligned}
0= & \delta\left(p^{2}-m^{2}\right)\left[p \cdot \nabla V+\frac{\hbar}{4}\left(\partial_{x}^{\alpha} \tilde{F}^{\mu \nu}\right) \partial_{p \alpha} \Sigma_{\mu \nu}\right] \\
& -\delta^{\prime}\left(p^{2}-m^{2}\right) \frac{\hbar}{2} F^{\mu \nu} p \cdot \nabla \Sigma_{\mu \nu}, \\
0= & \delta\left(p^{2}-m^{2}\right)\left[p \cdot \nabla n^{\mu}-F^{\mu \nu} n_{\nu}-\frac{\hbar}{2} p_{\nu}\left(\partial_{x}^{\alpha} \tilde{F}^{\mu \nu}\right) \partial_{p \alpha} V\right] \\
& +\delta^{\prime}\left(p^{2}-m^{2}\right) \hbar \tilde{F}^{\mu \nu} p_{\nu} p \cdot \nabla V .
\end{aligned}
$$

We note that the solution in Eqs. (2.4), the constraint (2.5), and the kinetic equations (2.7) are for massive fermions. In the massless case, the Wigner function also has a formal solution, and the corresponding chiral kinetic equations up to $\mathcal{O}(\hbar)$ can be derived. In the rest part of this paper, we will explicitly show that a smooth transition exists between the massive results (2.4)-(2.7) and the corresponding massless ones.

\section{REFERENCE-FRAME DEPENDENCE}

The components of the Wigner function in Eqs. (2.4) are obviously Lorentz covariant. The quantities $V(x, p)$ and $\Sigma^{\mu \nu}(x, p)$ have clear physical meanings of a fermion number (vector charge) density and dipole-moment tensor, respectively. Furthermore, in obtaining Eqs. (2.4), it is not necessary to introduce any additional reference-frame vector because one can always work in the comoving frame of the massive particle. However, this is not the case for massless fermions: the massless solutions in Refs. [40-43] are inevitably reference-frame dependent. Such a reference frame controls the way of decomposing a four vector such as $\mathcal{V}^{\mu}(x, p)$ and $\mathcal{A}^{\mu}(x, p)$ into one part parallel to $p^{\mu}$ and the other part perpendicular to $p^{\mu}$. It also controls the way of decomposing an antisymmetric tensor such as $\Sigma^{\mu \nu}(x, p)$ into an "electric" part and a "magnetic" part. Thus, in order to find a smooth transition to the massless fermions, we need to first introduce a reference frame to the Wigner-function solutions for massive fermions. We will achieve this by decomposing the dipolemoment tensor into the "electric" and a "magnetic" parts. In the Appendix A, we discuss an alternative, but equivalent, way to introduce the reference frame.

It is well-known that the electromagnetic field tensor $F^{\mu \nu}$ can be decomposed into the electric field $E^{\mu}=F^{\mu \nu} u_{\nu}$ and the magnetic field $B^{\mu}=(1 / 2) \epsilon^{\mu \nu \alpha \beta} u_{\nu} F_{\alpha \beta}$ as

$$
F^{\mu \nu}=E^{\mu} u^{\nu}-E^{\nu} u^{\mu}+\epsilon^{\mu \nu \alpha \beta} u_{\alpha} B_{\beta},
$$

where $u^{\mu}$ is an arbitrary timelike vector which is normalized as $u^{\mu} u_{\mu}=1$. The electric and magnetic field four vectors are all spacelike, i.e., $u_{\mu} E^{\mu}=u_{\mu} B^{\mu}=0$. Thus, in the comoving frame of $u^{\mu}, E^{\mu}$ and $B^{\mu}$ only have the spatial components, or they become three vectors. Such a decomposition depends on the choice of $u^{\mu}$, but $F^{\mu \nu}$ is independent of $u^{\mu}$. Similar to $F^{\mu \nu}$, the dipole-moment tensor $\Sigma^{\mu \nu}$ can also be decomposed as

$$
\Sigma^{\mu \nu}=\mathcal{E}^{\mu} u^{\nu}-\mathcal{E}^{\nu} u^{\mu}-\epsilon^{\mu \nu \alpha \beta} u_{\alpha} \mathcal{M}_{\beta},
$$

where $\mathcal{E}^{\mu}=\Sigma^{\mu \nu} u_{\nu}$ and $\mathcal{M}^{\mu}=-(1 / 2) \epsilon^{\mu \nu \alpha \beta} u_{\nu} \Sigma_{\alpha \beta}$. Note that both $\mathcal{E}^{\mu}$ and $\mathcal{M}^{\mu}$ depend on $u^{\mu}$, while $\Sigma^{\mu \nu}$ does not. In the comoving frame of $u^{\mu}$, we can identify $\mathcal{E}^{\mu}$ as the electric dipole moment and $\mathcal{M}^{\mu}$ as the magnetic dipole moment, respectively. Their physical meanings can be clearly seen by contracting $F_{\mu \nu}$ and $\Sigma^{\mu \nu}$,

$$
-\frac{1}{2} F_{\mu \nu} \Sigma^{\mu \nu}=-\mathcal{E}_{\mu} E^{\mu}-\mathcal{M}_{\mu} B^{\mu}
$$


which is the interaction energy of the dipole moments in the electromagnetic field.

Due to the constraint (2.5), the electric dipole moment $\mathcal{E}^{\mu}$ is not an independent degree of freedom. Substituting Eq. (3.2) into Eq. (2.5), up to $\mathcal{O}(\hbar)$, we obtain

$$
\begin{aligned}
& \delta\left(p^{2}-m^{2}\right)\left[(u \cdot p) \mathcal{E}^{\mu}-p_{\nu} \mathcal{E}^{\nu} u^{\mu}-\epsilon^{\mu \nu \alpha \beta} p_{\nu} u_{\alpha} \mathcal{M}_{\beta}-\frac{\hbar}{2} \nabla^{\mu} V\right] \\
& \quad=0 .
\end{aligned}
$$

Then contracting this equation with $u_{\mu}$, one obtains a constraint for the electric dipole moment,

$$
\delta\left(p^{2}-m^{2}\right)\left(p_{\mu} \mathcal{E}^{\mu}+\frac{\hbar}{2} u_{\mu} \nabla^{\mu} V\right)=0
$$

Inserting Eq. (3.5) back into Eq. (3.4), we find a general expression for the electric dipole moment,

$$
\begin{aligned}
\mathcal{E}^{\mu}= & \frac{\hbar}{2(u \cdot p)}\left(g^{\mu \nu}-u^{\mu} u^{\nu}\right) \nabla_{\nu} V \\
& +\frac{1}{u \cdot p} \epsilon^{\mu \nu \alpha \beta} p_{\nu} u_{\alpha} \mathcal{M}_{\beta}+\left(p^{2}-m^{2}\right) \mathcal{C}^{\mu},
\end{aligned}
$$

where $\mathcal{C}^{\mu}$ is an arbitrary function, which should be nonsingular for on shell momentum with $p^{2}=m^{2}$. We demand that $u_{\mu} \mathcal{C}^{\mu}=0$ because $u_{\mu} \mathcal{E}^{\mu}=0$. Inserting Eq. (3.6) back into Eq. (3.2), we find that $\Sigma^{\mu \nu}$ can be determined by $\mathcal{M}^{\mu}$, $V$, and $\mathcal{C}^{\mu}$,

$$
\begin{aligned}
\Sigma^{\mu \nu}= & -\frac{1}{u \cdot p} \epsilon^{\mu \nu \alpha \beta} p_{\alpha} \mathcal{M}_{\beta}+\frac{\hbar}{2(u \cdot p)}\left(u^{\nu} \nabla^{\mu}-u^{\mu} \nabla^{\nu}\right) V \\
& +\left(p^{2}-m^{2}\right)\left(u^{\nu} \mathcal{C}^{\mu}-u^{\mu} \mathcal{C}^{\nu}\right),
\end{aligned}
$$

where we have used the Schouten identity,

$u^{\mu} \epsilon^{\nu \alpha \beta \gamma}+u^{\nu} \epsilon^{\alpha \beta \gamma \mu}+u^{\alpha} \epsilon^{\beta \gamma \mu \nu}+u^{\beta} \epsilon^{\gamma \mu \nu \alpha}+u^{\gamma} \epsilon^{\mu \nu \alpha \beta}=0$.

In Eq. (3.7), we have an unspecified term, which depends on $\mathcal{C}^{\mu}$. However, since the forms of the solutions (2.4) do not change under the transformation with arbitrary $\delta \Sigma^{\mu \nu}[61]$,

$$
\begin{aligned}
\Sigma^{\mu \nu} & \rightarrow \Sigma^{\prime \mu \nu}=\Sigma^{\mu \nu}+\left(p^{2}-m^{2}\right) \delta \Sigma^{\mu \nu}, \\
V & \rightarrow V^{\prime}=V-\frac{\hbar}{2} F^{\mu \nu} \delta \Sigma_{\mu \nu},
\end{aligned}
$$

we can choose $\mathcal{C}^{\mu}=0$ without loss of generality. When the electromagnetic field vanishes, all components of the Wigner function are proportional to $\delta\left(p^{2}-m^{2}\right)$, and thus, the contribution from $\mathcal{C}^{\mu}$ is zero by its prefactor $\left(p^{2}-m^{2}\right)$.
We further define the projection operators,

$\Delta^{\mu \nu} \equiv g^{\mu \nu}-u^{\mu} u^{\nu}, \quad \Xi^{\mu \nu} \equiv g^{\mu \nu}-u^{\mu} u^{\nu}+\frac{p^{\langle\mu\rangle} p^{\langle\nu\rangle}}{(u \cdot p)^{2}-p^{2}}$,

where $p^{\langle\mu\rangle} \equiv \Delta^{\mu \nu} p_{\nu}$. The operator $\Delta^{\mu \nu}$ projects a four vector to the direction perpendicular to $u^{\mu}$, while the operator $\Xi^{\mu \nu}$ projects it to the direction perpendicular to both $u^{\mu}$ and $p^{\mu}$. Then, we define the transverse magnetic dipole-moment vector as follows:

$$
\mathcal{M}_{\perp}^{\mu} \equiv \Xi^{\mu \nu} \mathcal{M}_{\nu}=\mathcal{M}^{\mu}-\frac{u \cdot p}{(u \cdot p)^{2}-p^{2}} p^{\langle\mu\rangle} A,
$$

where $A$ is defined as

$$
A \equiv-\frac{\mathcal{M} \cdot p}{u \cdot p}
$$

and will be identified as the axial-charge density. Using $A$ and $\mathcal{M}_{\perp}^{\mu}$, the dipole-moment tensor $\Sigma^{\mu \nu}$ in Eq. (3.7) can be put into the form,

$$
\begin{aligned}
\Sigma^{\mu \nu}= & \frac{u \cdot p}{(u \cdot p)^{2}-p^{2}} \epsilon^{\mu \nu \alpha \beta} p_{\alpha} u_{\beta} A-\frac{1}{u \cdot p} \epsilon^{\mu \nu \alpha \beta} p_{\alpha} \mathcal{M}_{\perp \beta} \\
& +\frac{\hbar}{2(u \cdot p)}\left(u^{\nu} \nabla^{\mu}-u^{\mu} \nabla^{\nu}\right) V .
\end{aligned}
$$

With the spin three-vector $\mathbf{n}$ in a specific frame, the particle is called longitudinally (transversely) polarized if $\mathbf{n}$ is parallel (perpendicular) to $\mathbf{p}$. Any other polarization state can be expressed as a superposition of a longitudinal polarization state and a transverse one. Generalizing such a three-dimensional decomposition to a four-dimensional one, we can decompose the axial vector $n^{\mu}$ as

$$
n^{\mu}=(u \cdot p) u^{\mu} \frac{u \cdot n}{u \cdot p}+n_{\|} p^{\langle\mu\rangle}+n_{\perp}^{\mu}
$$

where $n_{\perp}^{\mu}=\Xi^{\mu \nu} n_{\nu}$. In the comoving frame of $u^{\mu}$, $u \cdot n / u \cdot p$ is identified as the axial-charge density, $n_{\|}$is the longitudinal spin polarization, and $n_{\perp}^{\mu}$ is the transverse spin polarization. Since $n^{\mu}$ satisfies the constraint $p \cdot n=0$, $n_{\|}$can be expressed by

$$
n_{\|}=\frac{(u \cdot p)(u \cdot n)}{(u \cdot p)^{2}-p^{2}}
$$

Inserting $\Sigma^{\mu \nu}$ into Eq. (2.6), we obtain another form of $n^{\mu}$, 


$$
\begin{aligned}
n^{\mu}= & p^{\mu} A+\frac{p^{2}}{u \cdot p} \mathcal{M}^{\mu}+\frac{\hbar}{2(u \cdot p)} \epsilon^{\mu \nu \alpha \beta} p_{\nu} u_{\alpha} \nabla_{\beta} V \\
= & p^{\mu} A+\frac{p^{2}}{(u \cdot p)^{2}-p^{2}} p^{\langle\mu\rangle} A+\frac{p^{2}}{u \cdot p} \mathcal{M}_{\perp}^{\mu} \\
& +\frac{\hbar}{2(u \cdot p)} \epsilon^{\mu \nu \alpha \beta} p_{\nu} u_{\alpha} \nabla_{\beta} V .
\end{aligned}
$$

Making a comparison between Eq. (3.14) and Eq. (3.16), we find $A=u \cdot n / u \cdot p$ is the axial-charge density, while the transverse polarization is

$$
n_{\perp}^{\mu}=\frac{p^{2}}{u \cdot p} \mathcal{M}_{\perp}^{\mu}+\frac{\hbar}{2(u \cdot p)} \epsilon^{\mu \nu \alpha \beta} p_{\nu} u_{\alpha} \nabla_{\beta} V
$$

In Sec. IV, we will show that the second term of $n_{\perp}^{\mu}$ is the OAM of a wave packet, also known as the side-jump term $[41,42,44,49]$. This term remains in the massless limit if we keep the wave packet description for massless particles. We will also prove that $\mathcal{M}_{\perp}^{\mu} \propto 1 / m$ in a smallmass limit, which agrees with our knowledge about the magnetic moment and is divergent at the zero mass limit. However, $\mathcal{M}_{\perp}^{\mu}$ always comes with $p^{2}$ in $n_{\perp}^{\mu}$, so its contribution, $p^{2} \mathcal{M}_{\perp}^{\mu} \propto m$, smoothly goes towards zero for vanishing $m$.

\section{WIGNER FUNCTION FOR A WAVE PACKET}

In this section, we introduce the wave packet description of a single particle state into the Wigner function. For simplicity, we neglect the external electromagnetic field. The Wigner function is then given by

$$
\begin{aligned}
W(x, p)= & \int \frac{d^{4} q}{(2 \pi \hbar)^{6}} \sum_{s s^{\prime}} \exp \left(\frac{i}{\hbar} q \cdot x\right) \delta\left(p^{2}+\frac{1}{4} q^{2}-m^{2}\right) \delta(p \cdot q) \sqrt{\left|p^{0}+\frac{1}{2} q^{0}\right|\left|p^{0}-\frac{1}{2} q^{0}\right|} \\
& \times \theta\left(p^{0}\right) \bar{u}_{s}\left(\mathbf{p}+\frac{1}{2} \mathbf{q}\right) \otimes u_{s^{\prime}}\left(\mathbf{p}-\frac{1}{2} \mathbf{q}\right)\left\langle\Omega\left|\hat{a}_{\mathbf{p}+\frac{1}{2} \mathbf{q}, s}^{\dagger} \hat{a}_{\mathbf{p}-\frac{1}{2} \mathbf{q}, s^{\prime}}\right| \Omega\right\rangle .
\end{aligned}
$$

Here we only keep the contribution from particles and neglect that from antiparticles. The discussion for antiparticles can be similarly handled. The particle's spinors are denoted as $u_{s}(\mathbf{p})$ and $\bar{u}_{s}(\mathbf{p})$ with $s$ denoting the spin state. The state $|\Omega\rangle$ is assumed to take a wave packet form,

$$
|\Omega\rangle=\left|\mathbf{p}_{0}, s_{0},+\right\rangle_{\text {wp }}=\frac{1}{N} \int \frac{d^{3} \mathbf{p}^{\prime}}{(2 \pi \hbar)^{3}} \exp \left[-\frac{\left(\mathbf{p}^{\prime}-\mathbf{p}_{0}\right)^{2}}{4 \sigma_{p}^{2}}+\frac{i}{\hbar} p^{\prime} \cdot x_{0}\right] a_{\mathbf{p}^{\prime}, s_{0}}^{\dagger}|0\rangle,
$$

where the normalization constant $N=\sqrt{\left[\sigma_{p} /(\hbar \sqrt{2 \pi})\right]^{3}}$ ensures the unit condition $\langle\Omega \mid \Omega\rangle=1$ and $p^{\prime \mu}=\left(E_{\mathbf{p}^{\prime}} \equiv\right.$ $\left.\sqrt{\mathbf{p}^{\prime 2}+m^{2}}, \mathbf{p}^{\prime}\right)$ is the on shell four momentum. Such a wave packet is the Gaussian type with the center momentum $\mathbf{p}_{0}$ and center position $\mathbf{x}_{0}$ at time $t_{0}$. The spin state of the wave packet is labeled as $s_{0}$ in a given spin quantization direction $\mathbf{n}_{0}$. The momentum width of the wave packet is $\sigma_{p}$. In general, we assume the wave packet to be narrow enough in momentum space so that $\sigma_{p} \ll\left|\mathbf{p}_{0}\right|$. Thus, we can assume that the expectation value $\left\langle\Omega\left|\hat{a}_{\mathbf{p}+\frac{1}{2} \mathbf{q}, s}^{\dagger} \hat{a}_{\mathbf{p}-\frac{1}{2} \mathbf{q}, s^{\prime}}\right| \Omega\right\rangle$ vanish for a large $|\mathbf{q}| \gg \sigma_{p}$. One can then treat $\mathbf{q}$ as a small expansion variable in Eq. (4.1) except for $\exp (i q \cdot x / \hbar)$ and $\delta(p \cdot q)$. Due to the existence of $\exp (i q \cdot x / \hbar)$, $\mathbf{q}$ can be replaced by $i \hbar \nabla_{\mathbf{x}}$; in this sense, the expansion in $\mathbf{q}$ is equivalent to the gradient expansion. The leading and next-to-leading order terms in the Wigner function read

$$
W(x, p)=\frac{1}{(2 \pi \hbar)^{3}} \delta\left(p^{2}-m^{2}\right) \theta\left(p^{0}\right) \sum_{s s^{\prime}}\left[\bar{u}_{s}(\mathbf{p}) \otimes u_{s^{\prime}}(\mathbf{p})+\hbar \mathcal{U}_{s s^{\prime}} \cdot i \nabla_{\mathbf{x}}\right] f_{s s^{\prime}}(x, \mathbf{p}),
$$

where the distribution function is defined as

$$
f_{s s^{\prime}}(x, \mathbf{p})=\int \frac{d^{4} q}{(2 \pi \hbar)^{3}} \delta\left(q^{0}-\frac{\mathbf{p} \cdot \mathbf{q}}{E_{\mathbf{p}}}\right) \exp \left(\frac{i}{\hbar} q \cdot x\right)\left\langle\Omega\left|\hat{a}_{\mathbf{p}+\frac{1}{2} \mathbf{q}, s}^{\dagger} \hat{a}_{\mathbf{p}-\frac{1}{2} \mathbf{q}, s^{\prime}}\right| \Omega\right\rangle
$$

The three vector $\mathcal{U}_{s s^{\prime}}$ in Eq. (4.3) contains momentum derivatives of the spinors,

$$
\mathcal{U}_{s s^{\prime}} \equiv \frac{1}{2}\left\{\left[\nabla_{\mathbf{p}} \bar{u}_{s}(\mathbf{p})\right] \otimes u_{s^{\prime}}(\mathbf{p})-\bar{u}_{s}(\mathbf{p}) \otimes\left[\nabla_{\mathbf{p}} u_{s^{\prime}}(\mathbf{p})\right]\right\}
$$


which represents a Berry connection in Dirac space, and its explicit form is calculated in Appendix B. With Eq. (4.2), we can give the analytical form of the distribution function as

$$
f_{s s^{\prime}}(x, \mathbf{p})=(2 \pi \hbar)^{3} V_{0}(x, \mathbf{p}) \delta_{s s_{0}} \delta_{s^{\prime} s_{0}},
$$

where the Gaussian type distribution function $V_{0}(x, p)$ is given by

$$
V_{0}(x, \mathbf{p})=\frac{8}{(2 \pi \hbar)^{3}} \exp \left\{-\frac{\left(\mathbf{p}-\mathbf{p}_{0}\right)^{2}}{2 \sigma_{p}^{2}}-\frac{2 \sigma_{p}^{2}}{\hbar^{2}}\left[\left(\mathbf{x}-\mathbf{x}_{0}\right)-\frac{\mathbf{p}}{E_{\mathbf{p}}}\left(t-t_{0}\right)\right]^{2}\right\} .
$$

One can read in the above form of $V_{0}(x, p)$, the center momentum $\mathbf{p}_{0}$ and the center position $\mathbf{x}_{0}+\left(t-t_{0}\right) \mathbf{p} / E_{\mathbf{p}}$. Note that the center position moves with the velocity $\mathbf{p} / E_{\mathbf{p}}$, reflecting the movement of the wave packet. Since we have $\nabla_{\mathbf{x}} V_{0} \propto\left[\left(\mathbf{x}-\mathbf{x}_{0}\right)-\mathbf{p} / E_{\mathbf{p}}\left(t-t_{0}\right)\right] V_{0}$, we can identify $\left(\mathbf{p} \times \nabla_{\mathbf{x}}\right) V_{0} \propto\left[\mathbf{p} \times\left(\mathbf{x}-\mathbf{x}_{0}\right)\right] V_{0}$ as the OAM of the wave packet. Inserting $f_{s s^{\prime}}(x, \mathbf{p})$ in (4.6) into the Wigner function in (4.3), we derive the scalar component of the Wigner function by taking the trace of $W$,

$$
\mathcal{F}=2\left[m-\frac{\hbar}{2\left(E_{\mathbf{p}}+m\right)} s_{0} \mathbf{n}_{0} \cdot\left(\mathbf{p} \times \nabla_{\mathbf{x}}\right)\right] V_{0}(x, \mathbf{p}) \delta\left(p^{2}-m^{2}\right) \theta\left(p^{0}\right),
$$

where $\mathbf{n}_{0}$ is the spin quantization direction, and the second term on the right-hand side is interpreted as a correction from the spin-orbital coupling. Equation (4.8) is given in the lab frame with $u^{\mu}=(1,0,0,0)$; a straightforward generalization to an arbitrary frame gives

$$
V(x, \mathbf{p})=2\left[1-\frac{\hbar}{2 m(u \cdot p+m)} s_{0} \epsilon^{\mu \nu \alpha \beta} u_{\mu} n_{0 \nu} p_{\alpha} \partial_{x \beta}\right] V_{0}(x, \mathbf{p}) .
$$

Analogously, we can derive the axial-vector component of the Wigner function,

$$
\begin{aligned}
& \mathcal{A}^{0}=2 s_{0} \mathbf{p} \cdot \mathbf{n}_{0} V_{0}(x, \mathbf{p}) \delta\left(p^{2}-m^{2}\right) \theta\left(p^{0}\right), \\
& \mathcal{A}=2\left[s_{0} m \mathbf{n}_{0}+s_{0} \frac{\mathbf{p} \cdot \mathbf{n}_{0}}{E_{\mathbf{p}}+m} \mathbf{p}-\frac{\hbar}{2\left(E_{\mathbf{p}}+m\right)} \mathbf{p} \times \nabla_{\mathbf{x}}\right] V_{0}(x, \mathbf{p}) \delta\left(p^{2}-m^{2}\right) \theta\left(p^{0}\right) .
\end{aligned}
$$

Comparing with Eq. (3.14) with the reference frame taken as the lab frame $u^{\mu}=(1,0,0,0)$, we obtain the axial-charge distribution $A$ and the transverse magnetic dipole moment as

$$
\begin{aligned}
A(x, \mathbf{p}) & =2 s_{0} \frac{\mathbf{p} \cdot \mathbf{n}_{0}}{E_{\mathbf{p}}} V_{0}(x, \mathbf{p}), \\
\mathcal{M}_{\perp}(x, \mathbf{p}) & =\frac{2}{m}\left[s_{0} E_{\mathbf{p}}\left(\mathbf{n}_{0}-\frac{\mathbf{p} \cdot \mathbf{n}_{0}}{E_{\mathbf{p}}^{2}-m^{2}} \mathbf{p}\right)+\frac{\hbar}{2\left(E_{\mathbf{p}}+m\right)} \mathbf{p} \times \nabla_{\mathbf{x}}\right] V_{0}(x, \mathbf{p}) .
\end{aligned}
$$

Generalizing them to an arbitrary frame with a general $u^{\mu}$, we obtain

$$
\begin{aligned}
A & =-2 s_{0} \frac{p^{\alpha} \Delta_{\alpha \beta} n_{0}^{\beta}}{u \cdot p} V_{0}(x, p), \\
\mathcal{M}_{\perp}^{\mu} & =\frac{2}{m}\left[s_{0}(u \cdot p) \Xi_{\nu}^{\mu} n_{0}^{\nu}+\frac{\hbar}{2(u \cdot p+m)} \epsilon^{\mu \nu \alpha \beta} u_{\nu} p_{\alpha} \partial_{x \beta}\right] V_{0}(x, p),
\end{aligned}
$$

where the projection operators $\Delta^{\mu \nu}$ and $\Xi^{\mu \nu}$ are defined in Eq. (3.10). We see that the transverse magnetic dipole moment consists of two parts: one is the particle's spin $s_{0} n_{0}^{\mu}$ as the intrinsic degrees of freedom, and the other is from the spatial derivative of the distribution that can be identified as the OAM of the wave packet.
If there are many particles in the system, the calculation of the Wigner function in the wave packet representation is straightforward: we first calculate the each particle's Wigner function, and then sum over all particles to obtain the total Wigner function. At equilibrium, this can also be achieved by replacing $|\Omega\rangle$ with the thermal state. 
It can be easily verified that the small-mass behaviors of $V, A$, and $\mathcal{M}_{\perp}^{\mu}$ are

$$
\begin{aligned}
V & =\mathcal{O}\left(m^{-1}\right)+\mathcal{O}(1, m, \cdots), \\
A & =\mathcal{O}(1)+\mathcal{O}\left(m, m^{2}, \cdots\right), \\
\mathcal{M}_{\perp}^{\mu} & =\mathcal{O}\left(m^{-1}\right)+\mathcal{O}(1, m, \cdots) .
\end{aligned}
$$

In the next section, we will show that, in the Wigner function and the kinetic equation, the divergent parts of $V$ and $\mathcal{M}_{\perp}^{\mu}$ at $m \rightarrow 0$ will either be canceled or be suppressed by a factor $m^{2}$.

\section{CONNECTION BETWEEN MASSIVE AND MASSLESS KINETIC EQUATIONS}

For massless fermions, only the vector and axial-vector components of the Wigner function are relevant to the kinetic equations. In this section, we focus on these components and show how to smoothly reproduce the massless formula from the massive ones from Eq. (2.4). We also show how to recover the CKT in the massless limit from the kinetic equations for massive fermions.

\section{A. Wigner function components}

Using the transverse part of the magnetic moment $\mathcal{M}_{\perp}^{\mu}$ and the axial-charge distribution $A$, the axial-vector component of Wigner function is obtained by substituting Eq. (3.16) into the axial-vector component in Eq. (2.4),

$$
\begin{aligned}
\mathcal{A}^{\mu}= & {\left[(u \cdot p) u^{\mu}+\frac{(u \cdot p)^{2}}{(u \cdot p)^{2}-m^{2}} p^{\langle\mu\rangle}\right] A \delta\left(p^{2}-m^{2}\right) } \\
& +\frac{\hbar}{2(u \cdot p)} \epsilon^{\mu \nu \alpha \beta} p_{\nu} u_{\alpha}\left(\nabla_{\beta} V\right) \delta\left(p^{2}-m^{2}\right) \\
& +\hbar \tilde{F}_{\mu \nu} p^{\nu} V \delta^{\prime}\left(p^{2}-m^{2}\right)+\frac{m^{2}}{u \cdot p} \mathcal{M}_{\perp}^{\mu} \delta\left(p^{2}-m^{2}\right) .
\end{aligned}
$$

In the massless limit, we find that the last term vanish because $\mathcal{M}_{\perp}^{\mu} \propto m^{-1}$, while the remaining parts agree with the massless result in Refs. [40-43].

On the other hand, inserting the dipole-moment tensor (3.7) into the vector component of the Wigner function in Eq. (2.4) gives

$$
\begin{aligned}
\mathcal{V}^{\mu}= & p^{\mu} V \delta\left(p^{2}-m^{2}\right)+\hbar \tilde{F}^{\mu \nu} p_{\nu} A \delta^{\prime}\left(p^{2}-m^{2}\right)+m^{2} \frac{\hbar}{u \cdot p} \tilde{F}^{\mu \nu} \mathcal{M}_{\nu} \delta^{\prime}\left(p^{2}-m^{2}\right) \\
& -\frac{\hbar}{2} \delta\left(p^{2}-m^{2}\right) \epsilon^{\mu \nu \alpha \beta} p_{\alpha} \nabla_{\nu}\left(\frac{1}{u \cdot p} \mathcal{M}_{\beta}\right),
\end{aligned}
$$

where we have used the Schouten identity (3.8). It is not easy to see the small-mass-behavior of the last term, so we choose to rewrite it using the following relation:

$$
\begin{aligned}
\epsilon^{\mu \nu \alpha \beta} p_{\alpha} \nabla_{\nu}\left(\frac{1}{u \cdot p} \mathcal{M}_{\beta}\right)= & -\frac{1}{u \cdot p} p^{\mu} u_{\rho} \epsilon^{\nu \alpha \beta \rho} p_{\alpha} \nabla_{\nu}\left(\frac{1}{u \cdot p} \mathcal{M}_{\beta}\right)-\frac{p^{2}}{u \cdot p} u_{\rho} \epsilon^{\beta \rho \mu \nu} \nabla_{\nu}\left(\frac{1}{u \cdot p} \mathcal{M}_{\beta}\right) \\
& -\frac{1}{u \cdot p} u_{\rho} p_{\alpha} \epsilon^{\alpha \beta \rho \mu} p^{\nu} \nabla_{\nu}\left(\frac{1}{u \cdot p} \mathcal{M}_{\beta}\right)-\frac{1}{u \cdot p} u_{\rho} p_{\alpha} p^{\beta} \epsilon^{\rho \mu \nu \alpha} \nabla_{\nu}\left(\frac{1}{u \cdot p} \mathcal{M}_{\beta}\right),
\end{aligned}
$$

which can be proved using the Schouten identity (3.8). Since the spin polarization $n^{\mu}$ satisfies the generalized BMT equation, i.e., the second equation in Eq. (2.7), we obtain the following kinetic equation for the magnetic moment by replacing $n^{\mu}$ with Eq. (3.16):

$$
\delta\left(p^{2}-m^{2}\right) p^{\nu} \nabla_{\nu}\left(\frac{1}{u \cdot p} \mathcal{M}^{\mu}\right)=\left[\frac{1}{u \cdot p} F^{\mu \nu} \mathcal{M}_{\nu}-\frac{1}{m^{2}} p^{\mu}\left(p^{\nu} \nabla_{\nu} A\right)\right] \delta\left(p^{2}-m^{2}\right)+\mathcal{O}(\hbar) .
$$

Inserting the above relation and Eq. (5.3) into Eq. (5.2), we obtain

$$
\begin{aligned}
\mathcal{V}^{\mu}= & p^{\mu} \tilde{V} \delta\left(p^{2}-m^{2}\right)+\hbar \tilde{F}^{\mu \nu} p_{\nu} A \delta^{\prime}\left(p^{2}-m^{2}\right) \\
& -\frac{\hbar}{2(u \cdot p)} \epsilon^{\mu \nu \alpha \beta} u_{\nu} p_{\alpha}\left(\nabla_{\beta} A\right) \delta\left(p^{2}-m^{2}\right) \\
& +m^{2}\left\{\frac{\hbar}{2(u \cdot p)} \epsilon^{\mu \nu \alpha \beta} u_{\nu}\left[\nabla_{\alpha}\left(\frac{1}{u \cdot p} \mathcal{M}_{\beta}\right)\right] \delta\left(p^{2}-m^{2}\right)+\frac{\hbar}{u \cdot p} \tilde{F}^{\mu \nu} \mathcal{M}_{\nu} \delta^{\prime}\left(p^{2}-m^{2}\right)\right\},
\end{aligned}
$$

where we have redefined the distribution as 


$$
\tilde{V} \equiv V+\frac{\hbar}{2(u \cdot p)} \epsilon^{\alpha \beta \rho \sigma} u_{\alpha} p_{\beta} \nabla_{\rho}\left(\frac{1}{u \cdot p} \mathcal{M}_{\sigma}\right)
$$

In the massless limit, $\mathcal{V}^{\mu}$ in Eq. (5.5) smoothly reproduces the result in Refs. [40-43]. We note that $\mathcal{M}_{\perp}^{\mu} \propto m^{-1}$ and $V \propto m^{-1}$ so that $\tilde{V}$ seems to be divergent for small $m$. However, the divergent part of $V$ cancels exactly that of $\mathcal{M}^{\mu}$ leaving a finite $\tilde{V}$ in massless limit. In fact, taking a Gaussian wave packet as an example, as shown in Sec. IV, we obtain

$$
\begin{aligned}
\tilde{V}= & \frac{1}{2}\left[1+\frac{\hbar}{2(u \cdot p)(u \cdot p+m)} s_{0} \epsilon^{\mu \nu \alpha \beta} u_{\mu} n_{0 \nu} p_{\alpha} \partial_{x \beta}\right] \\
& \times V_{0}(x, \mathbf{p}),
\end{aligned}
$$

which is regular in $m \rightarrow 0$ limit, where we have assumed a constant $u^{\mu}$ and vanishing electromagnetic field for simplicity and $V_{0}(x, \mathbf{p})$ is the distribution for the considered wave packet given in Eq. (4.7).
We note that the reference-frame four vector $u^{\mu}$ can generally be a local vector in phase space, i.e., $u^{\mu}$ can be a function of $\left\{x^{\mu}, p^{\mu}\right\}$. Especially, if we take $u^{\mu}=p^{\mu} / m$, we would have $\mathcal{M}^{\mu}=n^{\mu} / m$ and $A=0$, so $\mathcal{A}^{\mu}$ and $\mathcal{V}^{\mu}$ in Eqs. (5.1), (5.5) recover their forms in Eq. (2.4).

\section{B. Kinetic equations}

In the previous subsection, we have discussed the vector and axial-vector components of the Wigner function. Since these components can smoothly recover their forms in the massless case, it is natural that the corresponding kinetic equations (2.7) can reproduce the CKT when $m \rightarrow 0$. In this subsection, we explicitly show this.

In order to separate the kinetic equation for the axialcharge density $A$, we first contract the generalized BMT equation (2.7) with $u_{\mu}$ and then substitute $n^{\mu}$ with the expression in Eq. (3.16). After a long but straightforward calculation and using the Schouten identity (3.8), we finally arrive at

$$
\begin{aligned}
0= & \left(p^{\mu} \nabla_{\mu} A\right) \delta\left(p^{2}-m^{2}\right)+\delta^{\prime}\left(p^{2}-m^{2}\right)\left[\frac{\hbar}{u \cdot p} \tilde{F}^{\mu \nu} u_{\mu} p_{\nu}\left(p^{\alpha} \nabla_{\alpha} V\right)\right] \\
& +\delta\left(p^{2}-m^{2}\right)\left[\frac{\hbar}{2} \epsilon^{\mu \nu \alpha \beta} p_{\nu}\left(\nabla_{\mu} \frac{u_{\alpha}}{u \cdot p}\right)\left(\nabla_{\beta} V\right)+\frac{\hbar}{2(u \cdot p)} p_{\mu} u_{\nu}\left(\partial_{x \alpha} \tilde{F}^{\mu \nu}\right)\left(\partial_{p}^{\alpha} V\right)\right] \\
& -\delta\left(p^{2}-m^{2}\right) \frac{m^{2}}{(u \cdot p)^{2}}\left[\left(p \cdot \nabla u_{\mu}-F_{\mu \nu} u^{\nu}\right) \mathcal{M}^{\mu}+\frac{\hbar}{2} \epsilon^{\mu \nu \alpha \beta}\left(\nabla_{\mu} V\right) u_{\nu} \nabla_{\alpha} u_{\beta}\right] .
\end{aligned}
$$

Since $\mathcal{O}\left(\hbar^{2}\right)$ terms are truncated throughout this paper, one can replace $V$ in the above equation with $\tilde{V}$. Meanwhile, the kinetic equation for the distribution $\tilde{V}$ can be derived from the generalized Boltzmann equation in Eq. (2.7) by substituting the dipole-moment tensor $\Sigma^{\mu \nu}$ with the expression (3.7). However, a simpler way is to directly act $\nabla_{\mu}$ on the reference-frame dependent $\mathcal{V}^{\mu}$ in Eq. (5.5). Properly using the Schouten identity (3.8) and the properties of delta functions,

$$
\begin{aligned}
& x \delta^{\prime}(x)=-\delta(x), \\
& x \delta^{\prime \prime}(x)=-2 \delta^{\prime}(x),
\end{aligned}
$$

we derive the kinetic equation for $\tilde{V}$ as follows:

$$
\begin{aligned}
0= & \left(p^{\mu} \nabla_{\mu} \tilde{V}\right) \delta\left(p^{2}-m^{2}\right)+\delta^{\prime}\left(p^{2}-m^{2}\right)\left[\frac{\hbar}{u \cdot p} \tilde{F}^{\mu \nu} u_{\mu} p_{\nu}\left(p^{\alpha} \nabla_{\alpha} A\right)\right] \\
& +\delta\left(p^{2}-m^{2}\right)\left[\frac{\hbar}{2} \epsilon^{\mu \nu \alpha \beta} p_{\nu}\left(\nabla_{\mu} \frac{u_{\alpha}}{u \cdot p}\right)\left(\nabla_{\beta} A\right)+\frac{\hbar}{2(u \cdot p)} p_{\mu} u_{\nu}\left(\partial_{x \alpha} \tilde{F}^{\mu \nu}\right)\left(\partial_{p}^{\alpha} A\right)\right] \\
& +\delta\left(p^{2}-m^{2}\right) m^{2}\left\{\frac{\hbar}{2} \epsilon^{\mu \nu \alpha \beta}\left(\nabla_{\mu} \frac{u_{\nu}}{u \cdot p}\right)\left[\nabla_{\alpha}\left(\frac{1}{u \cdot p} \mathcal{M}_{\beta}\right)\right]-\frac{\hbar}{2(u \cdot p)} u_{\mu}\left(\partial_{x \alpha} \tilde{F}^{\mu \nu}\right)\left[\partial_{p}^{\alpha}\left(\frac{1}{u \cdot p} \mathcal{M}_{\nu}\right)\right]\right\} \\
& +\delta^{\prime}\left(p^{2}-m^{2}\right) m^{2} \frac{\hbar}{u \cdot p} \tilde{F}^{\mu \nu} u_{\mu}\left[p^{\alpha} \nabla_{\alpha}\left(\frac{1}{u \cdot p} \mathcal{M}_{\nu}\right)\right] .
\end{aligned}
$$

In the massless limit, Eqs. (5.8) and (5.10) agree exactly with the results in Refs. [40-43]. Due to the chiral symmetry, equations for $A$ and $\tilde{V}$ have dual forms in the massless case. However, the mass corrections in Eq. (5.8) and (5.10) have very different forms. 
As we have discussed in Sec. III, $A$ and $\mathcal{M}_{\perp}^{\mu}$ are two independent variables in describing spin polarization of massive fermions. Now we have the kinetic equation for $A$ in (5.8). The kinetic equation for $\mathcal{M}_{\perp}^{\mu}$ can be derived from the generalized BMT equation (2.7) by substituting $n^{\mu}$ with the expression (3.16). The resulting equation is complicated, which we would not show here. This equation is, however, always accompanied with $m^{2}$ factor and becomes redundant in the massless limit. As a result, the kinetic equations, which describe the 4 spin d.o.f. of massive fermions, reduce to chiral kinetic equations describing 2 spin d.o.f. of massless fermions.

\section{SUMMARY}

We show how to smoothly connect the kinetic theories with the spin degree of freedom for massive and massless fermions. The Wigner-function components and the kinetic equations are expressed in a reference-frame dependent form. The reference frame is introduced as the freedom to decompose the dipole-moment tensor into an electric dipole-moment vector and a magnetic dipole-moment one. Meanwhile, the spin polarization is decomposed into an axial-charge distribution, a longitudinal polarization, and a transverse polarization. Here, a longitudinal (transverse) vector refers to the one that is parallel (orthogonal) to the three momentum in the reference frame. The axialcharge distribution is obtained by projecting the spin polarization onto the direction of the reference frame four vector. We find a straightforward relation between the longitudinal polarization and the axial-charge distribution. Thus, a minimum set of functions for describing massive fermions is the fermion distribution $V(x, p)$, the axialcharge distribution $A(x, p)$, and the transverse part of the magnetic dipole-moment $\mathcal{M}_{\perp}^{\mu}(x, p)$. By carefully calculating these functions through the Wigner function in the wave packet representation, we find their small-mass behaviors: $V \sim \mathcal{O}\left(m^{-1}\right), \mathcal{M}_{\perp}^{\mu} \sim \mathcal{O}\left(m^{-1}\right)$, and $A \sim \mathcal{O}(1)$.

With these small-mass behaviors, we can extract the mass corrections in the vector and axial vector components of the Wigner function as well as their corresponding kinetic equations. By turning off the mass corrections, we can smoothly recover their forms in the massless case. Therefore, the CKT can be obtained by a smooth transition from the kinetic theory for massive fermions with spin. We note that the collision terms are not included in this paper, which are reserved for a future work. We expect that the side-jump effect can also arise naturally in the collision terms for massive fermions following the same line when taking the massless limit.

\section{ACKNOWLEDGMENTS}

The authors thank X.-Y. Guo, Y.-C. Liu, E. Speranza, and $\mathrm{S}$. $\mathrm{Pu}$ for enlightening discussions. X.-L.S. and Q.W. are supported by the 973 program under Grant
No. 2015CB856902 and by NSFC under Grant No. 11535012. X.-G.H is supported by NSFC under Grants No. 11535012 and No. 11675041.

Note added.-Recently, we became aware of a related study [72].

\section{APPENDIX A: AN ALTERNATIVE WAY TO INTRODUCE $u^{\mu}$ IN $\mathcal{V}^{\mu}$ AND $\mathcal{A}^{\mu}$}

In Sec. III, we introduce the reference frame by decomposing the dipole-moment tensor into the electric and magnetic components. In this Appendix, we adopt an alternative way to introduce the reference frame.

In Ref. [63], general forms of $\mathcal{V}^{\mu}$ and $\mathcal{A}^{\mu}$ up to $\mathcal{O}(\hbar)$ have been derived,

$$
\begin{aligned}
& \mathcal{V}^{\mu}= \delta\left(p^{2}-m^{2}\right)\left(p^{\mu} f+\frac{\hbar}{2 u \cdot p} \epsilon^{\mu \nu \rho \sigma} u_{\nu} \nabla_{\rho} n_{\sigma}\right) \\
&+\hbar \tilde{F}^{\mu \nu}\left(n_{\nu}-u_{\nu} \frac{p \cdot n}{p \cdot u}\right) \delta^{\prime}\left(p^{2}-m^{2}\right), \\
& \mathcal{A}^{\mu}=n^{\mu} \delta\left(p^{2}-m^{2}\right)+\hbar \tilde{F}^{\mu \nu} p_{\nu} f \delta^{\prime}\left(p^{2}-m^{2}\right),
\end{aligned}
$$

where the reference-frame vector $u^{\mu}$ is introduced when solving one of the equations of motion for the Wigner-function components (see, e.g., Refs. [60-63]): $(\hbar / 2)\left(\nabla_{\mu} \mathcal{A}_{\nu}-\nabla_{\nu} \mathcal{A}_{\mu}\right)=\epsilon_{\mu \nu \rho \sigma} p^{\rho} \mathcal{V}^{\sigma}+\mathcal{O}\left(\hbar^{2}\right)$. Here, $f$ is the vector charge density, which is identical to $V$ in the main text at $\mathcal{O}(1)$ but can differ from $V$ at $\mathcal{O}(\hbar)$. In fact, $\mathcal{V}^{\mu}$ and $\mathcal{A}^{\mu}$ in Eqs. (A1) and (A2) are equivalent to those in Eq. (2.4) because the solutions are invariant under transformations (3.9) and $n_{\mu} \rightarrow n_{\mu}+\left(p^{2}-m^{2}\right) \delta n_{\mu}, f \rightarrow f+$ $\hbar \tilde{F}^{\mu \nu} u_{\mu} \delta n_{\nu} / u \cdot p$. Substituting Eqs. (A1) and (A2) into the relation (see, e.g., Refs. [60-63]) $m \mathcal{S}_{\mu \nu}=(\hbar / 2) \times$ $\left(\nabla_{\mu} \mathcal{V}_{\nu}-\nabla_{\nu} \mathcal{V}_{\mu}\right)-\epsilon_{\mu \nu \rho \sigma} p^{\rho} \mathcal{A}^{\sigma}+\mathcal{O}\left(\hbar^{2}\right)$, we obtain

$\mathcal{S}^{\mu \nu}=m\left[\Sigma^{\mu \nu} \delta\left(p^{2}-m^{2}\right)-\hbar F^{\mu \nu} f \delta^{\prime}\left(p^{2}-m^{2}\right)\right]+\mathcal{O}\left(\hbar^{2}\right)$.

In the above, we have defined the dipole-moment tensor as

$\Sigma^{\mu \nu}=-\frac{1}{u \cdot p} \epsilon^{\mu \nu \alpha \beta} p_{\alpha} \mathcal{M}_{\beta}+\frac{\hbar}{2(u \cdot p)}\left(u^{\nu} \nabla^{\mu}-u^{\mu} \nabla^{\nu}\right) f$,

where

$$
\begin{gathered}
\mathcal{M}^{\mu}=\frac{1}{m^{2}}\left[\left(n^{\mu}-p^{\mu} A\right) p \cdot u-\frac{\hbar}{2} \epsilon^{\mu \nu \alpha \beta} p_{\nu} u_{\alpha} \nabla_{\beta} f\right], \\
A=\frac{u \cdot n}{u \cdot p}=-\frac{\mathcal{M} \cdot p}{u \cdot p} .
\end{gathered}
$$


Comparing to Eq. (3.16) and noticing that $f=V+\mathcal{O}(\hbar)$, we realize that $\mathcal{M}^{\mu}$ we defined here is the magnetic dipole moment, and the reference-frame vector $u^{\mu}$ is equivalent to the one introduced in the main text. From Eq. (A5), we can reexpress $n^{\mu}$ in terms of $\mathcal{M}$ and $A$. By substituting $n^{\mu}$ into Eqs. (A1) and (A2), after some algebra, we recover Eqs. (5.1) and Eq. (5.5), but with

$$
\tilde{V} \equiv f-\frac{\hbar}{(u \cdot p)^{2}} \tilde{F}^{\mu \nu} u_{\mu} \mathcal{M}_{\nu}
$$

\section{APPENDIX B: MOMENTUM-DERIVATIVE OF WAVE FUNCTIONS}

In this Appendix, we will calculate the Berry connection $\mathcal{U}_{s s^{\prime}}=\frac{1}{2}\left\{\left[\nabla_{\mathbf{p}} \bar{u}_{s}(\mathbf{p})\right] \otimes u_{s^{\prime}}(\mathbf{p})-\bar{u}_{s}(\mathbf{p}) \otimes\left[\nabla_{\mathbf{p}} u_{s^{\prime}}(\mathbf{p})\right]\right\}$. Since we are considering massive fermions, we can express the wave function $u_{s}(\mathbf{p})$ as a Lorentz boost of the wave function in the rest frame $u_{s, \mathrm{ff}}$,

$$
u_{s}(\mathbf{p})=\Lambda_{\mathbf{p}} u_{s, \mathrm{rf}}
$$

In this way, all the momentum dependence is embedded in the transformation matrix $\Lambda_{p}$. The explicit form of $\Lambda_{p}$ is well-known and can be found in many textbooks,

$$
\Lambda_{\mathbf{p}}=\frac{1}{\sqrt{m}}\left(\begin{array}{cc}
\sqrt{p \cdot \sigma} & 0 \\
0 & \sqrt{p \cdot \bar{\sigma}}
\end{array}\right)
$$

where $\sigma^{\mu} \equiv(1, \boldsymbol{\sigma})$ and $\bar{\sigma}^{\mu} \equiv(1,-\boldsymbol{\sigma})$. After complicated but straightforward calculations, we obtain the following relation:

$$
\begin{aligned}
\boldsymbol{\Delta}_{\mathbf{p}} \equiv & \left(\nabla_{\mathbf{p}} \Lambda_{\mathbf{p}}\right) \Lambda_{\mathbf{p}}^{-1}=\frac{1}{2 m}\left[\gamma^{0} \boldsymbol{\gamma}-\frac{1}{E_{\mathbf{p}}\left(E_{\mathbf{p}}+m\right)} \mathbf{p}\left(\mathbf{p} \cdot \gamma^{0} \boldsymbol{\gamma}\right)\right] \\
& -\frac{i}{2 m\left(E_{\mathbf{p}}+m\right)} \mathbf{p} \times \gamma^{5} \boldsymbol{\gamma}^{0} \boldsymbol{\gamma} .
\end{aligned}
$$

Then the momentum derivatives of the wave functions $u_{s}(\mathbf{p})$ and $\bar{u}_{s}(\mathbf{p})$ are given by

$$
\begin{aligned}
& \nabla_{\mathbf{p}} u_{s}(\mathbf{p})=\boldsymbol{\Delta}_{\mathbf{p}} u_{s}(\mathbf{p}), \\
& \nabla_{\mathbf{p}} \bar{u}_{s}(\mathbf{p})=-\bar{u}_{s}(\mathbf{p}) \boldsymbol{\Delta}_{\mathbf{p}} .
\end{aligned}
$$

The Berry connection now takes the following form:

$$
\mathcal{U}_{s s^{\prime}}=-\frac{1}{2}\left[\bar{u}_{s}(\mathbf{p}) \boldsymbol{\Delta}_{\mathbf{p}} \otimes u_{s^{\prime}}(\mathbf{p})+\bar{u}_{s}(\mathbf{p}) \otimes \boldsymbol{\Delta}_{\mathbf{p}} u_{s^{\prime}}(\mathbf{p})\right] .
$$

[1] Z.-T. Liang and X.-N. Wang, Phys. Rev. Lett. 94, 102301 (2005); 96, 039901(E) (2006).

[2] B. Betz, M. Gyulassy, and G. Torrieri, Phys. Rev. C 76, 044901 (2007).

[3] F. Becattini, F. Piccinini, and J. Rizzo, Phys. Rev. C 77, 024906 (2008).

[4] V. Skokov, A. Yu. Illarionov, and V. Toneev, Int. J. Mod. Phys. A 24, 5925 (2009).

[5] V. Voronyuk, V. D. Toneev, W. Cassing, E. L. Bratkovskaya, V. P. Konchakovski, and S. A. Voloshin, Phys. Rev. C 83, 054911 (2011).

[6] W.-T. Deng and X.-G. Huang, Phys. Rev. C 85, 044907 (2012).

[7] L. McLerran and V. Skokov, Nucl. Phys. A929, 184 (2014).

[8] K. Tuchin, Phys. Rev. C 88, 024911 (2013).

[9] W.-T. Deng and X.-G. Huang, Phys. Lett. B 742, 296 (2015).

[10] H. Li, X.-1. Sheng, and Q. Wang, Phys. Rev. C 94, 044903 (2016).

[11] W.-T. Deng and X.-G. Huang, Phys. Rev. C 93, 064907 (2016).

[12] Y. Jiang, Z.-W. Lin, and J. Liao, Phys. Rev. C 94, 044910 (2016); 95, 049904(E) (2017).

[13] L.-G. Pang, H. Petersen, Q. Wang, and X.-N. Wang, Phys. Rev. Lett. 117, 192301 (2016).
[14] X.-G. Deng, X.-G. Huang, Y.-G. Ma, and S. Zhang, Phys. Rev. C 101, 064908 (2020).

[15] S. A. Voloshin, arXiv:nucl-th/0410089.

[16] R.-h. Fang, L.-g. Pang, Q. Wang, and X.-n. Wang, Phys. Rev. C 94, 024904 (2016).

[17] J.-H. Gao, S.-W. Chen, W.-t. Deng, Z.-T. Liang, Q. Wang, and X.-N. Wang, Phys. Rev. C 77, 044902 (2008).

[18] X.-G. Huang, P. Huovinen, and X.-N. Wang, Phys. Rev. C 84, 054910 (2011).

[19] F. Becattini, V. Chandra, L. Del Zanna, and E. Grossi, Ann. Phys. (Amsterdam) 338, 32 (2013).

[20] J.-j. Zhang, R.-h. Fang, Q. Wang, and X.-N. Wang, Phys. Rev. C 100, 064904 (2019).

[21] L. Adamczyk et al. (STAR Collaboration), Nature (London) 548, 62 (2017).

[22] J. Adam et al. (STAR Collaboration), Phys. Rev. C 98, 014910 (2018).

[23] Q. Wang, Nucl. Phys. A967, 225 (2017).

[24] X.-G. Huang, arXiv:2002.07549.

[25] F. Becattini and M. A. Lisa, arXiv:2003.03640.

[26] A. Vilenkin, Phys. Rev. D 22, 3080 (1980).

[27] D. E. Kharzeev, L. D. McLerran, and H. J. Warringa, Nucl. Phys. A803, 227 (2008).

[28] K. Fukushima, D. E. Kharzeev, and H. J. Warringa, Phys. Rev. D 78, 074033 (2008). 
[29] D. E. Kharzeev, J. Liao, S. A. Voloshin, and G. Wang, Prog. Part. Nucl. Phys. 88, 1 (2016).

[30] X.-G. Huang, Rep. Prog. Phys. 79, 076302 (2016).

[31] K. Hattori and X.-G. Huang, Nucl. Sci. Tech. 28, 26 (2017).

[32] J. Zhao and F. Wang, Prog. Part. Nucl. Phys. 107, 200 (2019).

[33] W. Li and G. Wang, arXiv:2002.10397.

[34] Y.-C. Liu and X.-G. Huang, Nucl. Sci. Tech. 31, 56 (2020).

[35] D. T. Son and N. Yamamoto, Phys. Rev. Lett. 109, 181602 (2012).

[36] D. T. Son and N. Yamamoto, Phys. Rev. D 87, 085016 (2013).

[37] M. A. Stephanov and Y. Yin, Phys. Rev. Lett. 109, 162001 (2012).

[38] J.-H. Gao, Z.-T. Liang, S. Pu, Q. Wang, and X.-N. Wang, Phys. Rev. Lett. 109, 232301 (2012).

[39] J.-W. Chen, S. Pu, Q. Wang, and X.-N. Wang, Phys. Rev. Lett. 110, 262301 (2013).

[40] Y. Hidaka, S. Pu, and D.-L. Yang, Phys. Rev. D 95, 091901 (2017).

[41] A. Huang, S. Shi, Y. Jiang, J. Liao, and P. Zhuang, Phys. Rev. D 98, 036010 (2018).

[42] J.-H. Gao, Z.-T. Liang, Q. Wang, and X.-N. Wang, Phys. Rev. D 98, 036019 (2018).

[43] Y.-C. Liu, L.-L. Gao, K. Mameda, and X.-G. Huang, Phys. Rev. D 99, 085014 (2019).

[44] Y. Hidaka, S. Pu, and D.-L. Yang, Phys. Rev. D 97, 016004 (2018).

[45] J.-h. Gao, S. Pu, and Q. Wang, Phys. Rev. D 96, 016002 (2017).

[46] S. Carignano, C. Manuel, and J. M. Torres-Rincon, Phys. Rev. D 98, 076005 (2018).

[47] S. Lin and A. Shukla, J. High Energy Phys. 06 (2019) 060.

[48] X.-G. Huang and A. V. Sadofyev, J. High Energy Phys. 03 (2019) 084.

[49] J.-Y. Chen, D. T. Son, M. A. Stephanov, H.-U. Yee, and Y. Yin, Phys. Rev. Lett. 113, 182302 (2014).

[50] J.-Y. Chen, D. T. Son, and M. A. Stephanov, Phys. Rev. Lett. 115, 021601 (2015).
[51] J.-h. Gao, J.-Y. Pang, and Q. Wang, Phys. Rev. D 100, 016008 (2019).

[52] S. Y. F. Liu, Y. Sun, and C. M. Ko, arXiv:1910.06774.

[53] F. Becattini and I. Karpenko, Phys. Rev. Lett. 120, 012302 (2018).

[54] J. Adam et al. (STAR Collaboration), Phys. Rev. Lett. 123, 132301 (2019).

[55] Z.-T. Liang and X.-N. Wang, Phys. Lett. B 629, 20 (2005).

[56] Y.-G. Yang, R.-H. Fang, Q. Wang, and X.-N. Wang, Phys. Rev. C 97, 034917 (2018).

[57] X.-L. Sheng, L. Oliva, and Q. Wang, Phys. Rev. D 101, 096005 (2020).

[58] S. Hess and L. Waldmann, Z. Naturforsch. Teil A 21, 1529 (1966).

[59] S. Hess and L. Waldmann, Z. Naturforsch. Teil A 23, 1893 (1968).

[60] J.-H. Gao and Z.-T. Liang, Phys. Rev. D 100, 056021 (2019).

[61] N. Weickgenannt, X.-L. Sheng, E. Speranza, Q. Wang, and D. H. Rischke, Phys. Rev. D 100, 056018 (2019).

[62] K. Hattori, Y. Hidaka, and D.-L. Yang, Phys. Rev. D 100, 096011 (2019).

[63] Y.-C. Liu, K. Mameda, and X.-G. Huang, arXiv:2002 .03753 .

[64] D.-L. Yang, K. Hattori, and Y. Hidaka, J. High Energy Phys. 07 (2020) 070 .

[65] Z. Wang, X. Guo, S. Shi, and P. Zhuang, Phys. Rev. D 100, 014015 (2019).

[66] N. Mueller and R. Venugopalan, Phys. Rev. D 96, 016023 (2017).

[67] N. Mueller and R. Venugopalan, Phys. Rev. D 97, 051901 (2018).

[68] E. P. Wigner, Ann. Math. 40, 149 (1939); Nucl. Phys. B, Proc. Suppl. 6, 9 (1989).

[69] Y. S. Kim and E. P. Wigner, J. Math. Phys. (N.Y.) 28, 1175 (1987).

[70] Y. S. Kim and E. P. Wigner, J. Math. Phys. (N.Y.) 31, 55 (1990).

[71] D. Vasak, M. Gyulassy, and H. T. Elze, Ann. Phys. (N.Y.) 173, 462 (1987).

[72] X. Guo, arXiv:2005.00228. 